\title{
Self Activation Recognition System
}

\author{
Kritika Balihar \\ Assistant Professor \\ BPIT, Delhi, India \\ Naina Singh \\ B.Tech Scholar \\ BPIT, Delhi, India
}

\author{
Priya Tendwal \\ B.Tech Scholar \\ BPIT, Delhi, India \\ Juhee Kumari \\ $\mathrm{B}$,Tech Scholar \\ BPIT, Delhi, India
}

\begin{abstract}
Automatic face recognition technologies have made many improvements in saving time. So, it should be convenient for the schools and universities or other organization, as nowadays everyone is in a hurry so, this can save time. Substantiate of this attendance identification system is a remarkable subject in system control in computer communication. Here in face recognition project various technologies and algorithms have used which captures the human faces precisely and fast. This identification system tries to develop a class attendance system as it manual attendance system is time consuming. Adapting digital attendance management system can change how you manage your workforce.
\end{abstract}

\section{Keywords}

Face recognition, automatic attendence, face detection, image processing

\section{INTRODUCTION}

Presence of a student in a class is virtually important for an educational organization. By the traditional method, which is used in earlier days that is a monotonous task in many organizations. It is also a concern for faculties to mark attendance manually which might take 5-7 minutes of entire session. This procedure is a time taking and there is also some chances of proxy of students. Therefore, many organization started to install many other technologies for recording attendance like cloud support, advances analytics. With the smart application of digital assets, attendance system could be a boon-both for organization and student turning once complex process into an easy- to- monitor and straight forward activity.

\section{LITERATURE SURVEY}

In the paper author proposed that different types of face detection for detecting faces in different pose. Detecting face in different pattern based on techniques. Basic pattern for detecting face is nose, eyes, hair, ears and some time it based on tone of skin [1]. In the paper, authors have designed and implemented an attendance system which uses iris biometrics. Initially, the attendant were asked to register their details along with their unique iris structure. At the time of attendance, the system automatically took class attendance by capturing the image of each attendee, recognizing their iris, extract a feature of attendee and searching for a match in the created database. The prototype was web based [2].

This system based on real time face detection which improves the quality of image and it is fast to do process the detection. it converts the image in resolution $112 \times 92$. It would be around $11 \mathrm{~KB}$ of size.[3] Viola-Jones Algorithm has been used for face detection which detect human face using cascade classifier and also Adaboost algorithm it uses an iterative approach to learn from the mistakes of weak classifiers, and turn them into strong ones. and SMQT Features and SNOW Classifier Method,Local Binary Pattern (LBP). When it is compared to traditional attendance marking this system saves the time and also helps to monitor the students.[4]

\section{WORK FLOW}

Flow of our project is shown in figure 1, First, student is added in our record and then its face is detected using the haarcascade algo, then its record is saved in the database. Then proceed the same steps for every other student.

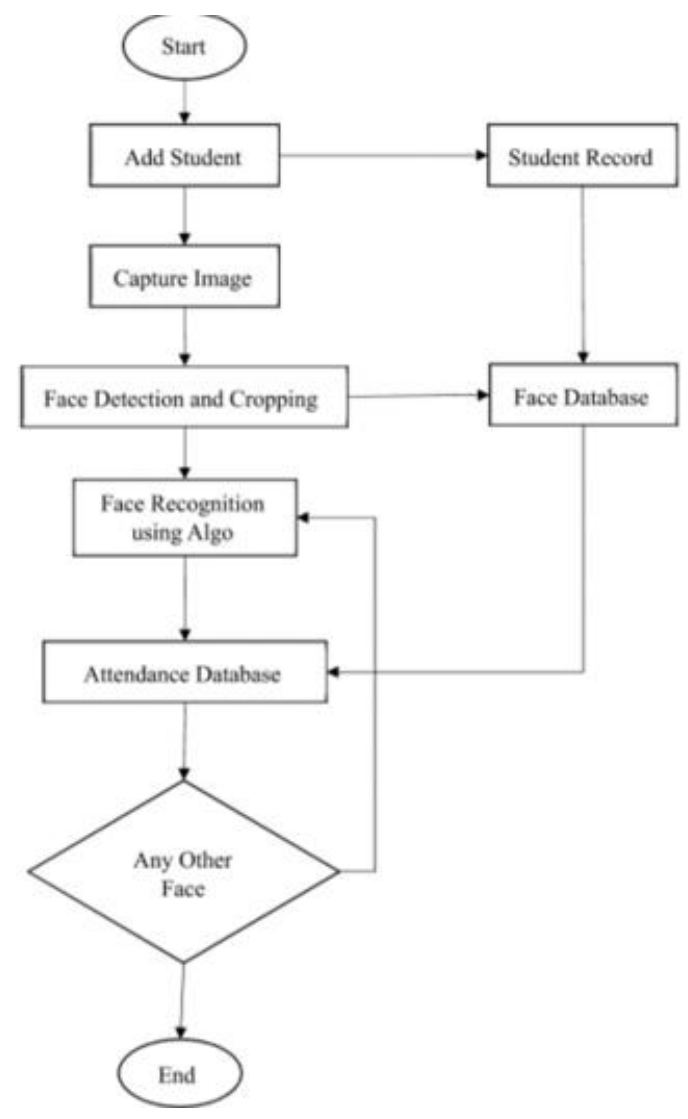

Fig 1: Architectural Flow Chart

\section{PROPOSED SYSTEM}

This recognition system going to capture the image of a person then it detect the face then it recognize it from the database that is provided to system and lastly it marks the attendance. In this system images are trained first so that it gives clear image and takes less space to store the image. 


\subsection{Training data}

From the database, images are captured from the camera or the webcam. For doing this, in python you have to install drivers from the math work website on the basis of type of camera you are using. Next, it is going to take 50-60 captures of each persons, so that it get higher percentage of accuracy and complete the purpose of this project. It can store the data in database distinguishing each and every person.

\subsection{Image Capture}

You need a good quality of camera in order to get better results. You can capture the picture manually by the camera. By training the images it can get better result and it saves the memory as well. But this works perfectly when there is a good light it won't work in less light.

\subsection{Face detection}

Facial extraction is a process where it extract face components like, nose, eyes, ears, mouth, etc. This project uses Haar feature based cascade classifier to detect object and its very important process of object detection method introduced by paul viola and michael jones.

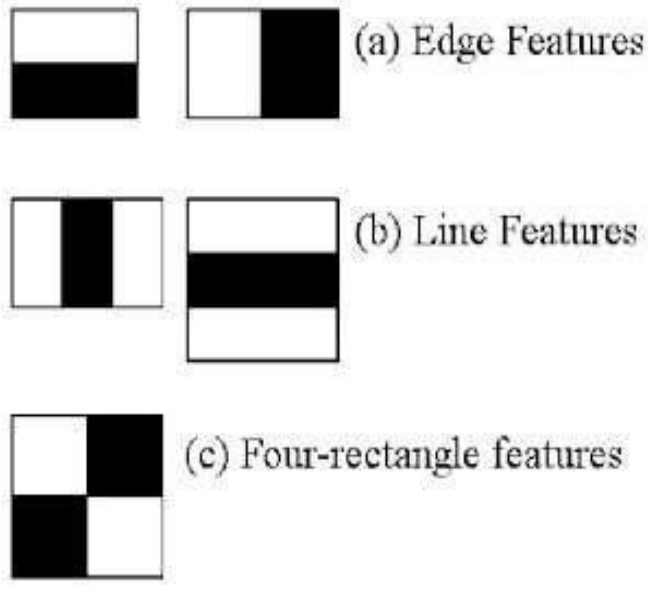

Fig 2: Feature extraction

\subsection{Face Recognition:}

Face could even be a complicated multidimensional visual model and developing a computational model for face recognition is difficult. The paper presents a technique for face recognition supported theory approach of coding and decoding the face image. Proposed methodology is interrelation of two stages- Face detection using Haar Based Cascade classifier and recognition using Principle Component analysis. Haar cascade frontal face default.xml is employed for detecting the face. Cascade is loaded using the cv2. Cascade Classifier function.

\section{FRONT END}

To implement this further student should be registered with their id and name.

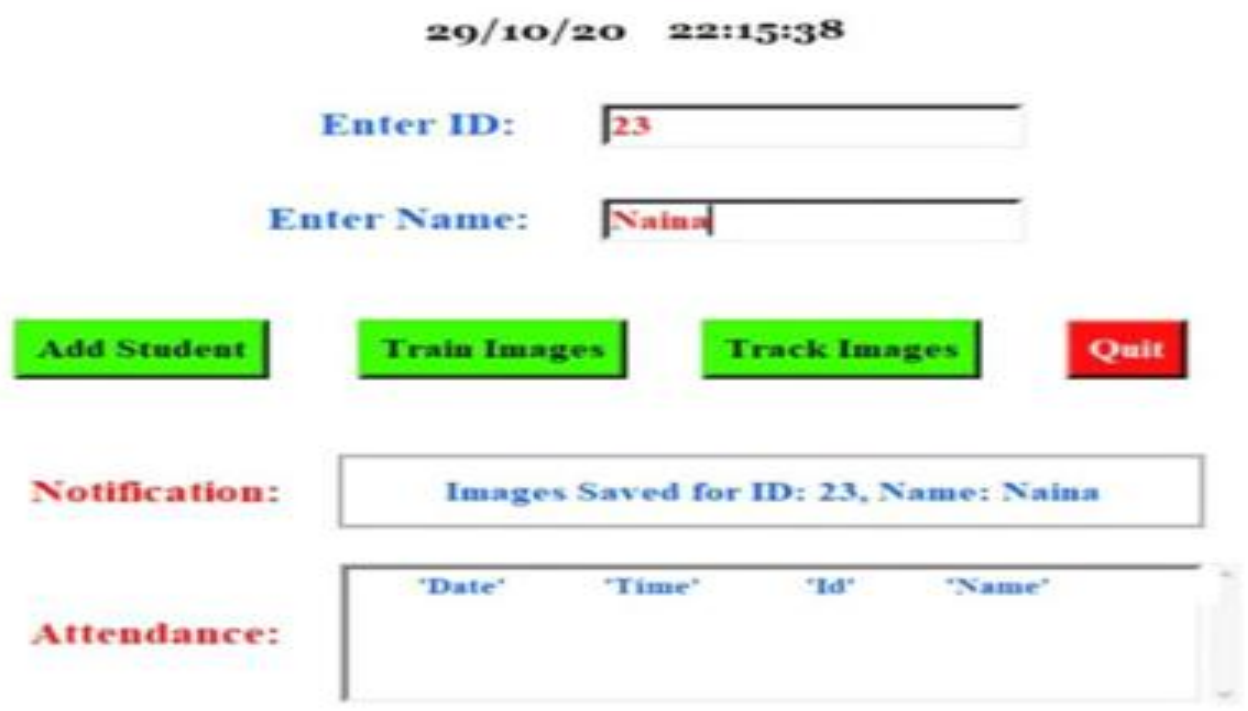

Fig 3: Register the Person into the Database

\subsection{Register the person:}

Now here project get the images of person, after capture the image it get stored in database of system, by taking 50 to 60 images it finds the most accurate image to store. 


\subsection{Capture and save images-}

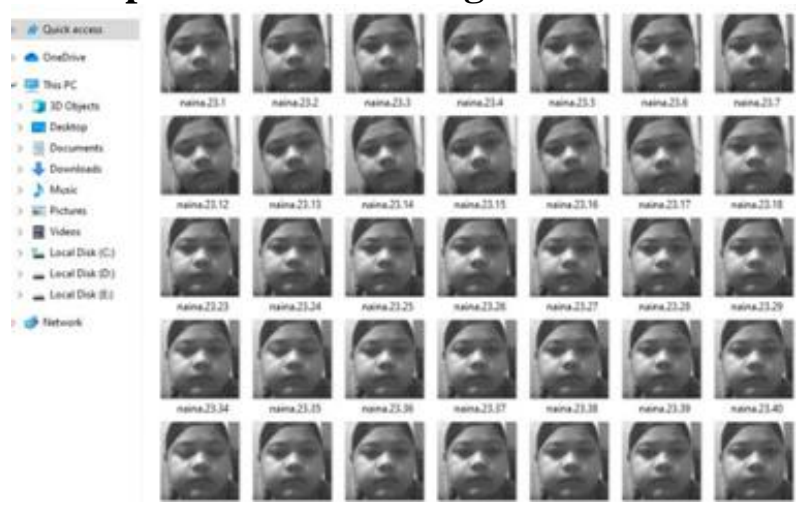

Fig 4: Capture and Save the Images in Database

Here training of images is done and it takes 50-60 images to find the accuracy of image, then it chooses the clear image.

\subsection{Attendance marking-}

Given data is stored in the database. With their ID number and name.

\begin{tabular}{|c|c|c|c|c|}
\hline$\Delta$ & A & $B$ & C & D \\
\hline 1 & ID & NAME & & \\
\hline 2 & 123 & Priya & & \\
\hline 3 & 125 & Juhee & & \\
\hline 4 & 345 & Naina & & \\
\hline 5 & 654 & Ankit & & \\
\hline 6 & & & & \\
\hline 7 & & & & \\
\hline 8 & & & & \\
\hline
\end{tabular}

\subsection{Recognizing the image}

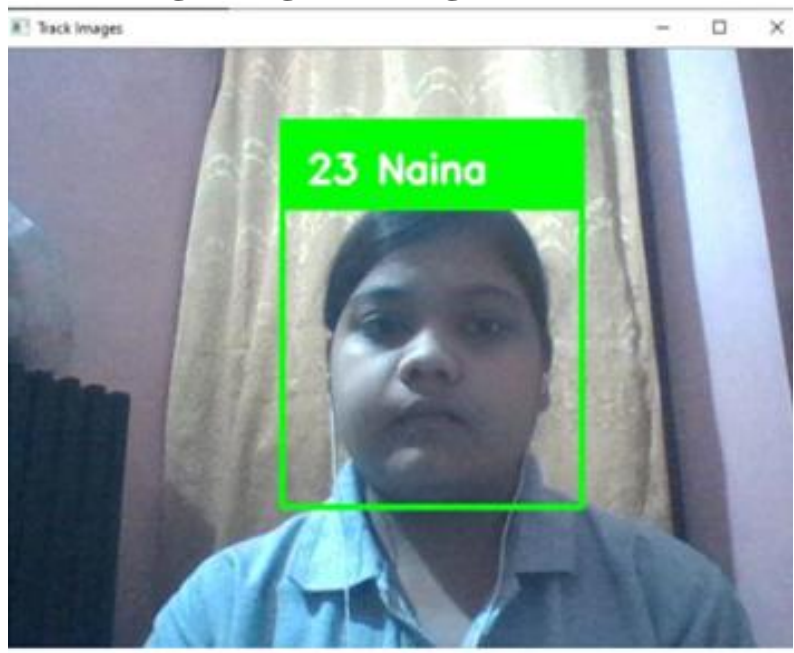

Fig 6: Recognizing Image and Show

After registered the id and name, it detects the person's face.

\section{CONCLUSION}

After examining different algorithms thoroughly and taking number of test of different condition images. It is observed that Haarcascade algorithm is the best algorithm in terms of speed and accuracy. The disadvantage of MTCNN algorithm is that it takes a lot of time. RGB and Haarcascade algo is used in this project to recognize the image. Component analysis method is used for face recognition. Student attendance have been marked by the help of recognized face of every single student and the data stored in attendance sheet. Attendance of every student that automatically marked by recognizing of the face which is present in database.

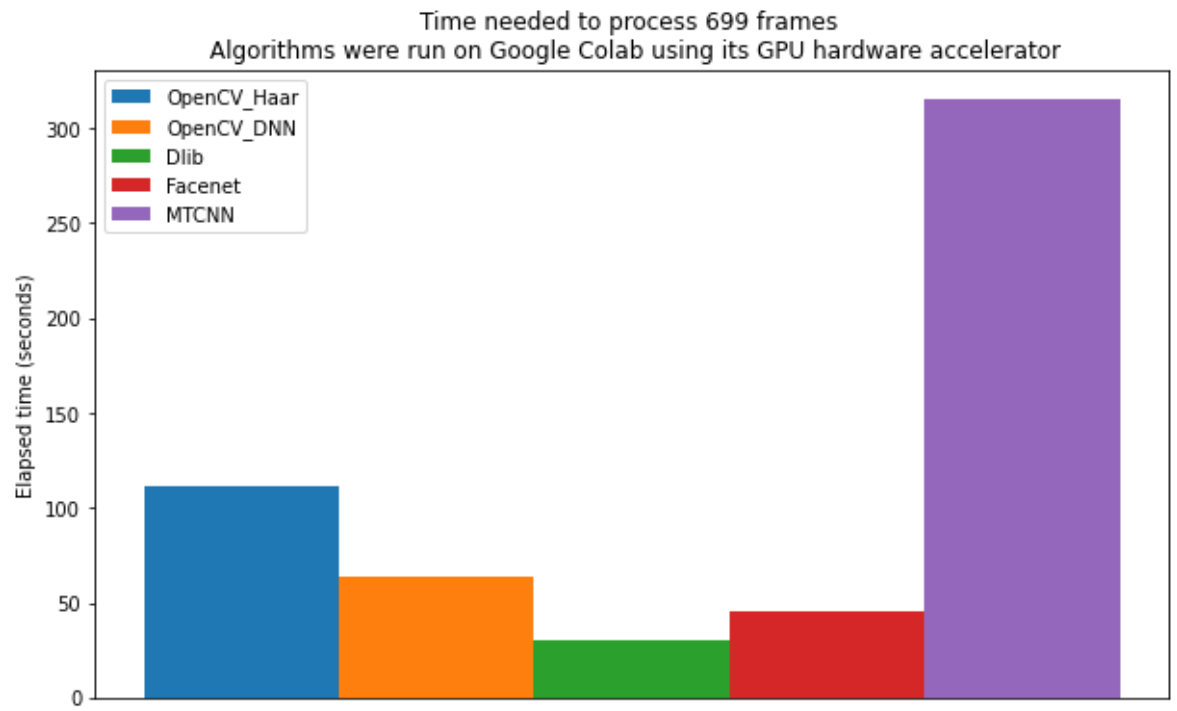

Fig 7: Comparison of face detecting algorithms

\section{ACKNOWLEDGMENTS}

Thanks to the Ms. Kritika, Assistant Professor, Department of Computer Science and Engineering, BPIT, Delhi who have contributed in development of the research paper.
8. REFERENCES

[1] Chakravorty, Pragnan (2018). "What is a Signal? [Lecture Notes]". IEEE Signal Processing Magazine. 35 (5): $175-177 . \quad$ Bibcode:2018ISPM...35..175C. doi:10.1109/MSP.2018.2832195.

[2] Okokpujie, Kennedy O., et al. "Design and 
implementation of a student attendance system using iris biometric recognition." 2017 International Conference on Computational Science and Computational Intelligence (CSCI). IEEE, 2017.

[3] Adam Schmidt, Andrzej Kasinski, "The Performance of the Haar Cascade Classifiers Applied to the Face and Eyes Detection", Computer Recognition Systems.

[4] Dan Wang, Rong Fu, Zuying Luo, "Classroom Attendance Auto-management Based on Deep Learning",Advances in Social Science, Education and Humanities Research, volume 123,ICESAME 2017.

[5] Attendance System based on Face Recognition Volume:05 Issue: 04, Apr-2018 Venkata Kalyan Polamarasetty, Muralidhar Reddy Dheeraj Ravi, Mahith Sai Madala https://www.irjet.net/archives/V5/i4/IRJETV5I41022.pdf

[6] A Study of Various Face Detection Methods, Ms.Varsha Gupta, Mr. Dipesh Sharma, ijarcce vol.3 https://www.ijarcce.com/upload/2014/may/IJARCCE7G $\% 2$

0\%20a\%20varsha\%20A\%20Study $\% 20$ of $\% 20$ Various $\% 2$ $0 \mathrm{~F}$ ace.pdf

[7] Attendance System Using Face Recognition and Class monitoring System, Arun Katara, Mr. Sudesh, V.Kolhe
http://www.ijritcc.org/download/browse/Volume5_Issues /Februar y_17_Volume_5_Issue_2/1489565866_15-032017.pdf

[8] Adam Schmidt, Andrzej Kasinski, "The Performance of the Haar Cascade Classifiers Applied to the Face and Eyes Detection", Computer Recognition Systems 2

[9] Automated Student Attendance Registering System Using Face Recognition by HIMANSHU MALLIK http://ethesis.nitrkl.ac.in/7301/1/AUTOMATED_Mallik_ 2015.pdf

[10] N.Sudhakar Reddy, M.V.Sumanth, S.Suresh Babu, "A Counterpart Approach to Attendance and Feedback System using Machine Learning Techniques",Journalof Emerging Technologies and Innovative Research (JETIR), Volume 5, Issue 12, Dec 2018.

[11] Prajakta Lad, Sonali More, Simran Parkhe, Priyanka Nikam, Dipalee Chaudhari, "Student Attendance System Using Iris Detection", IJARIIE-ISSN(O)-2395-4396, Vol-3 Issue-2 2017.

[12] N.Sudhakar Reddy, M.V.Sumanth, S.Suresh Babu, "A Counterpart Approach to Attendance and Feedback System using Machine Learning Techniques",Journal of Emerging Technologies and Innovative Research (JETIR), Volume 5, Issue 12, Dec 2018. 